\title{
Stabilizing High-order Delayed Systems with Minimum-phase Zeros Using Simple Controllers*
}

\author{
C. D. Vázquez Rosas ${ }^{1 *}$, J.F. Márquez Rubio ${ }^{1}$, B. del Muro Cuéllar ${ }^{1}$, \\ D.F. Novella Rodríguez ${ }^{2}$, O. Sename ${ }^{3}$, L. Dugard ${ }^{3}$ \\ ${ }^{1}$ Instituto Politécnico Nacional, ESIME Unidad Culhuacán, Av. Santa Ana 1000, \\ Coyoacán, 04430, CDMX, México \\ cdelectronics88@gmail.com (*Corresponding author),jfcomr23@yahoo.com.mx, bdelmuro@yahoo.com \\ ${ }^{2}$ Universidad de Monterrey, Departamento de Ingeniería, Ignacio Morones Prieto 4500, \\ 66238 San Pedro Garza García, N.L., México \\ dnovellar@gmail.mx \\ ${ }^{3}$ Univ. Grenoble Alpes, GIPSA-lab, Grenoble, France \\ olivier.sename@gipsa-lab.fr, luc.dugard@gipsa-lab.grenoble-inp.fr
}

\begin{abstract}
This paper analyses the stabilization and control of delayed processes systems containing minimum phase zeros. The high-order delayed process taken into consideration consists of: one unstable pole and $m$ minimum phase zeros. Sufficient conditions are provided in order to guarantee the existence of a stabilizing basic controllers: Proportional (P), Integral (I) and Derivative (D), i.e., P, PI, PD and PID controllers. The frequency domain approach is used to proof the main results. Additionally an optimal $H_{\infty}$ tuning of the controller is proposed. The adequateness of the obtained results are tested by simulation through the implementation of a continuously stirred-tank reactor model.
\end{abstract}

Keywords: Zeros, Unstable processes, Time-delay, Stabilization.

\section{Introduction}

Time-delay is a property present in many dynamical systems. When the delay is significant, compared with the main time constant in the process, it causes undesirable behaviours in a control system like poor performance, oscillations or even instability, (Kharitonov, 1998). This phenomenon can be found in many engineering systems such as: tele-operated process, chemical and biochemical engineering process, information and material transmission, (Wu et al., 2015). The particular problem of unstable systems with timedelay is usual in the chemical processes, such as continuously stirred-tank reactor (CSTR), (QingChang, 2006). Thus, the control and stabilization of delay processes becomes an interesting topic in which the explicit stability conditions for many unstable processes are not available yet.

Several solutions have been studied in order to face the stability problems for time-delay systems, for instance: (De Paor \& O'Malley, 1989) who

\footnotetext{
* This paper is an extended version of the work titled "Stability condition for unstable high-order system with minimumphase zeros plus time-delay", published in the Congreso Nacional de Control Automático (AMCA) 2017 (Vázquez et al. 2017). Employing a different method for the proof, the current paper presents improved sufficient conditions for P/PI controllers in order to stabilize the process taken into consideration. Another novel element is the sufficient condition to guarantee the existence of stabilizing PD/PID controllers. Finally the theory is employed in order to tune the parameters for the PI/PID controllers.
}

propose a modification to the original ZieglerNichols method, the Smith Predictor (SP), whose main objective is the elimination of the delay from the characteristic equation of the system. This last technique as originally presented can only be used in stable plants (Smith, 1957).

However, many authors have proposed modifications to the traditional SP to address the limitations of the original structure. In (Márquez et al., 2010), SP modifications are proposed to control and stabilize unstable first-order systems with time-delay. (Liu et al., 2005; Seshagiri et al., 2007) propose a control scheme based on a SP with two degree-of-freedom in order to stabilize the delayed systems.

A different proposal to stabilize time-delay systems is represented by the employment of simple controllers; for instance: (Silva et al., 2004) characterize the entire set of stabilizing Proportional - Integral Derivative controllers, (Xiang, 2006) investigates the stabilization of several delayed process with unstable poles by employing simple controllers. The mentioned works do not take into account the case when zeros are present in the transfer function of the process.

To address the case of controlling time-delay systems containing zeros in their transfer functions, (Kwak et al., 2000; Lee et al., 2010b) propose the use of $\mathrm{P}$ controllers for first and 
second-order unstable systems. An extended result is obtained in (Lee \& Wang, 2010) where the authors analyze a system with $n$ stable poles using different controllers. In these works, the authors establish explicit sufficient stabilizability conditions, but they only take into account the case when only one left half-plane (LHP) zero is considered in the transfer function of the original system. Nevertheless, in other recent research works, more general results addressing the case of systems with various zeros and poles can be found. In (Vázquez et al., 2017), sufficient conditions are provided to stabilize Single-Input-Single-Output (SISO) high-order delayed systems with only one unstable pole and containing $m$ real LHP zeros, using proportional $(\mathrm{P})$ controllers.

On the other hand, in practical cases, typical design requirements like the disturbance rejection, speed of response, and unmodeled dynamics must be ensured. In order to satisfy these requirements, $H_{\infty}$ control theory is a very useful tool to handle control systems with disturbances, uncertainties and parameter variations.

In this article, sufficient conditions are provided in order to guaranteed the stabilization of high-order delayed systems including $m$ real LHP zeros, by using Proportional (P)-Derivative (D)-Integral (I) controllers, i.e., P, PI, PD, and/or PID controllers. A frequency domain analysis is used to perform the formal proof. Then, a systematic design procedure using the $H_{\infty}$ theory is proposed in order to obtain the optimal gains for PI/PID controllers. The nonsmooth $H_{\infty}$ synthesis proposed in (Apkarian \& Noll, 2006) has been considered, which is an extension of the $H_{\infty}$ central design method (Doyle et al., 1989). The paper is organized in the following way: Section 1 gives an introduction of time-delay systems (definition, importance and challenges). The problem statement is the subject of Section 2. The purpose of the Section 3 is to present the preliminary results; a useful series of lemmas for the proofs of the main results. The proposed solution is presented in Section 4, which also includes the $H_{\infty}$ control design procedure. Numerical examples are presented to verify the design methods in Section 5 and finally in Section 6 , the conclusion summarizes the paper.

\section{Problem Statement}

Consider the next linear time-invariant (LTI) system, SISO with time-delay in the direct path:

$Y(s) / U(s)=H(s) e^{-\tau s}$, where $Y(s)$ is the output signal, $H(s)$ is the transfer function without delay, $U(s)$ is the input signal and $\tau>0$ is the time-delay.

Applying a control strategy $C(s)$ in the form:

$U(s)=C(s)[R(s)-Y(s)]$,

produces the next closed-loop system:

$\frac{Y(s)}{R(s)}=\frac{C(s) H(s) e^{-\tau s}}{1+C(s) H(s) e^{-\tau s}}$,

Take into account the process characterized by (1) with a $H(s)$ given by:

$$
H(s)=\frac{\prod_{l=1}^{m}\left(s+b_{l}\right)}{\left(s-a_{1}\right) \prod_{i=2}^{n+1}\left(s+a_{i}\right)},
$$

where $b_{l}, m, a_{1}, a_{i}, \tau>0, m \leq n+1$ and $n \geq 0$. For simplicity of developments and without loss of generality, a unitary open loop gain has been considered, in the transfer function. Now consider the output feedback given by (2) and the following four types of $C(s)$ compensators:

$$
\begin{aligned}
& C_{P}=k_{p} \\
& C_{P I}=k_{p}+\frac{k_{i}}{s} \\
& C_{P D}=k_{p}\left(s+k_{D}\right) \\
& C_{P I D}=k_{p}+\frac{k_{i}}{s}+k_{d} s
\end{aligned}
$$

Few works have addressed the case of unstable delayed system including zeros using these simple controllers. Recent research on the subject only considers the case of one zero in the original plant. For instance, stabilization problem for unstable time-delay systems with only one LHP zero by using simple controllers is considered in (Kwak et al., 2000; Lee et al., 2010b). The authors present the following result:

Lemma 1 (Kwak et al., 2000; Lee et al., 2010b). The process (1) with

$$
H(s)=(s+b) /(s-a)
$$

and $a, b, \tau>0$, is stabilized by using a $\mathrm{P}$ controller shown in (5) if and only if $a^{-1}>b^{-1}$ and $\tau<a^{-1}+b^{-1}$.

On the other hand, (Kwak et al., 2000; Xiang et al., 2007) demonstrate how to address the problem 
of stabilization for second order unstable timedelay system with one LHP zero, and present the next result:

Lemma 2 (Kwak et al., 2000; Xiang et al., 2007). The process (1) with

$H(s)=(s+b) /(s-a)(s+c)$

and $a, b, c, \tau>0$, is stabilized by using a $\mathrm{P}$ controller if $b^{-1}<\sqrt{a^{-1}+c^{-1}}$ and $\tau<a^{-1}+b^{-1}-c^{-1}$.

For high-order delayed systems with one minimum-phase zero, (Lee \& Wang, 2010) present the following result:

Lemma 3 (Lee \& Wang, 2010). The process (1) with:

$$
H(s)=(s+b) /(s-a) \prod_{i=1}^{n}\left(s+c_{i}\right)
$$

is stabilized by using a $\mathrm{P}$ controller

$$
\begin{aligned}
& \text { if } b^{-1}<\sqrt{a^{-1}+\sum_{i=1}^{n} c_{i}^{-1}} \text { and } \\
& \tau<a^{-1}+b^{-1}-\sum_{i=1}^{n} c_{i}^{-1}
\end{aligned}
$$

The principal objective of this article is to find a controller $C_{*}(s)$ (if any) such that it may stabilize the process (1) with $H(s)$ in the form shown in (4), in the closed-loop configuration.

Auxiliary open-loop transfer function $Q_{*}(s)$ can be formulated in the form:

$$
Q_{*}(s)=C_{*}(s) H(s) e^{-\tau s} \text {. }
$$

\section{Preliminary Results}

Now consider the open-loop transfer function (9) and the unitary feedback loop shown in (2), which result in the next closed-loop transfer function:

$$
\frac{Y(s)}{R(s)}=\frac{Q_{*}(s)}{1+Q_{*}(s)}
$$

The following results are related to the stability of the closed-loop transfer function (10). The Nyquist stability criterion is stated as first preliminary result.

Lemma 4 (Nyquist, 1932). The Nyquist stability criterion. Given the open-loop transfer function $Q_{*}(s)$ with $P^{+}$unstable poles, the associated closed-loop system shown in (10) is stable if and only if the Nyquist plot of $Q_{*}(\omega)$ encircles the critical point $(-1, j 0), P^{+}$times anticlockwise.

Lemma 5 (Lee et al., 2010; Xiang, 2006). Given the open-loop transfer function $Q_{*}(\omega), \lim M_{Q_{*}}(\omega)<1$ is a necessary condition for the stability of the associated closed loop system shown in (10), where $M_{Q_{*}}(\omega)$ denotes the magnitude of $Q_{*}(\omega)$.

Lemma 6 (Lee et al., 2010a; Xiang, 2006). If a process defined in (1), with $N(s) \neq 0$ can be stabilized by using a P controller, then it also can be stabilized by a PI controller. Similarly, stabilizablity achieved by means of a PD controller implies stabilizablity by employing a PID controller.

\section{Main Results}

This section presents the sufficient conditions in order to stabilize the delayed process given by (1) with $H(s)$ in the form shown in (4) by employing simple controllers.

\subsection{P/PI Controller}

Theorem 1. Consider the time-delay system given by (1) with $H(s)$ in the form shown in (4) and the P/PI controller given by (5)/(6), the associated closed-loop is stable if:

$\frac{\prod_{l=1}^{m} b_{l}}{\prod_{i=1}^{n+1} a_{i}}>\sqrt{\frac{\prod_{l=1}^{m}\left(\omega^{2}+b_{l}^{2}\right)}{\prod_{i=1}^{n+1}\left(\omega^{2}+a_{i}^{2}\right)}}, \forall \omega>0$

and

$\tau<\frac{1}{a_{1}}+\sum_{l=1}^{m} \frac{1}{b_{l}}-\sum_{i=2}^{n+1} \frac{1}{a_{i}}$

Let the main idea of the proof be introduced; a frequency domain analysis is used.

For $P$ controller $C_{p}$ and the system given by (1) with $H(s)$ in the form shown in (4), the open-loop frequency response is:

$$
\begin{aligned}
& Q_{p}(j \omega)=C_{p} H(j \omega) e^{-\tau j \omega} \\
& =k_{p} \frac{\prod_{l=1}^{m}\left(j \omega+b_{l}\right)}{\left(j \omega-a_{1}\right) \prod_{i=2}^{n+1}\left(j \omega+a_{i}\right)} e^{-\tau j \omega}
\end{aligned}
$$


The open- loop magnitude expression is:

$M_{Q_{p}}(\omega)=k_{p} \sqrt{\prod_{l=1}^{m}\left(\omega^{2}+b_{l}^{2}\right) / \prod_{i=1}^{n+1}\left(\omega^{2}+a_{i}^{2}\right)}$

And the associated open-loop phase is given by:

$\phi_{Q_{p}}(\omega)=-\left(\pi-\tan ^{-1} \frac{\omega}{a_{1}}\right)+\sum_{l=1}^{m} \tan ^{-1} \frac{\omega}{b_{l}}$

$-\sum_{i=2}^{n+1} \tan ^{-1} \frac{\omega}{a_{i}}-\omega \tau$

with its derivate as

$\frac{d \phi_{Q_{p}}(\omega)}{d \omega}=\frac{a_{1}}{a_{1}^{2}+\omega^{2}}+\sum_{l=1}^{m} \frac{b_{l}}{b_{l}^{2}+\omega^{2}}-$

$\sum_{i=2}^{n+1} \frac{a_{i}}{a_{i}^{2}+\omega^{2}}-\tau$

For the auxiliary system (13) and taking into account the Nyquist criteria, the system has $P^{+}=1$ unstable root. Then the Nyquist diagram should encircle once the point $(-1, \mathrm{j} 0)$ counterclockwise to guarantee the closed-loop stability.

As a first step, the phase of the system showing a growing stage for $\omega \approx 0$ (the required condition for the correct direction of the Nyquist plot) is analysed. Next, the expression for the magnitude of the system showing a decreasing magnitude for $\omega \approx 0$ assuring the correct direction of the Nyquist plot is examined. Finally, the high frequencies dynamic behaviour of the system is investigated in order to prevent the possibility of some additional encirclement around the critical point for $\omega \rightarrow \infty$.

In order to obtain the required encirclement for achieving closed-loop stability, it is necessary to have two intersections with the real negative axis of the s-plane, one intersection should occur into $[-\infty,-1]$ and the second one should appear into $[-1,0]$. That is: $M_{Q_{p}}\left(\omega_{c 1}\right)>1, \phi_{Q_{p}}\left(\omega_{c 1}\right)=-\pi$, $M_{Q_{p}}\left(\omega_{c_{2}}\right)<1, \phi_{Q_{p}}\left(\omega_{c_{2}}\right)=-\pi$.

where $\omega_{c_{1}}$ and $\omega_{c_{2}}$ are non-negative crossover frequencies and $\omega_{c_{1}}<\omega_{c_{2}}$. A desirable behaviour of the Nyquist and Bode plots is illustrated in Figures 1 and 2.

Furthermore, to ensure the mentioned encirclement, the behaviour of $M_{Q_{p}}$ and $\phi_{Q_{p}}$ are analyzed so as to complete the proof.

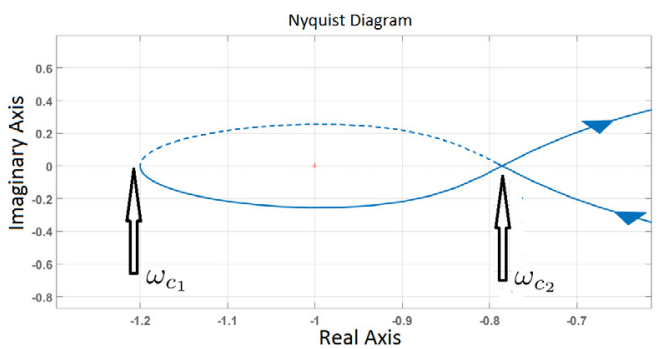

Figure 1. Nyquist diagram

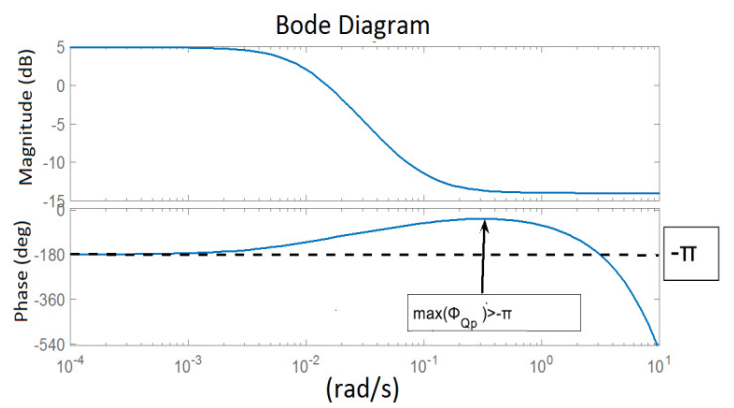

Figure 2. Bode diagram

Proof. Assume that the conditions shown in Theorem 1 are satisfied. To analyze the behaviour of the phase $\phi_{Q_{p}}$ given by (15), it should be taken into consideration that the phase plot starts at $\phi_{Q_{p}}(0)=-\pi$. Then, the first necessary intersection is obtained with $\phi_{Q_{p}}\left(\omega_{c_{1}}\right)=-\pi$, therefore $\omega_{c_{1}}=0$. To guarantee the desired direction of the Nyquist plot, as it is illustrated in Figure 2, the phase should be greater than $-\pi$, for some $\omega>0$ that is:

$\max \left(\phi_{Q_{p}}(\omega)\right)>-\pi$

In order to assure the condition (17), a growing of $\phi_{Q_{p}}(\omega)$ is necessary since $\phi_{Q_{p}}(0)=-\pi$, this implies $d \phi_{Q_{p}}(\omega) /\left.d \omega\right|_{\omega \rightarrow 0}>0$. In this way and taking into account $\omega_{c_{1}} \stackrel{\omega \rightarrow 0}{=} 0$ the following expression is evaluated:

$\left.\frac{d \phi_{Q_{p}}(\omega)}{d \omega}\right|_{\omega \rightarrow 0}=\frac{1}{a_{1}}+\sum_{l=1}^{m} \frac{1}{b_{l}}-\sum_{i=2}^{n+1} \frac{1}{a_{i}}-\tau>0$

Then, it can be established that under condition (12), there exists a positive $\omega$ such that $\max \left(\phi_{Q_{p}}(\omega)\right)>-\pi$. Analyzing the behavior of the phase $\phi_{Q_{p}}$ for high frequencies: $d \phi_{Q_{p}}(\omega) / d \omega=-\tau$. Then $\phi_{Q_{p}}(\omega)$ decreases for high frequencies. From the above developments, it can be observed that $\phi_{Q_{p}}$ increases from $\phi_{Q_{p}}\left(\omega_{c 1}\right)=-\pi$, after this, the phase 
decreases back to $-\pi$. This implies the second necessary intersection with $\phi_{Q_{p}}\left(\omega_{c_{2}}\right)=-\pi$, for some $\omega_{c_{2}}>0$. From this, it immediately follows that $\omega_{c_{1}}<\omega_{c_{2}}$.

Further on, the desired performance of $M_{Q_{p}}(\omega)$ is analyzed. As it is illustrated in Figure $1, M_{Q_{p}}$ should decrease for $\omega>0$. The value of $M_{Q_{p}}(\omega)$ with $\omega=0$ is $M_{Q_{p}}(0)=\prod_{l=1}^{m} b_{l} / \prod_{i=1}^{n+1} a_{i}$. Thus, to achieve the closed-loop stabilization and assuming that condition (11) is fulfilled, it can be assured that $M_{Q_{p}}(\omega)$ behaves like a decreasing function since $\omega=0$, getting the desired direction of the Nyquist diagram.

Following Lemma 5, it is also required to keep $\lim _{\omega \rightarrow \infty} M_{Q_{p}}(\omega)<1$ to prevent the possibility of $\underset{\omega \rightarrow \infty}{\omega \rightarrow m e}$ additional encirclement around the point $(-1, \mathrm{j} 0)$ for $\omega \rightarrow \infty$. If $m<n+1, Q_{p}(s)$ is a strictly proper system, then the behavior of $M_{Q_{p}}$ for high frequencies is: $M_{Q_{p}}(\omega)=0$. For $m=n+1$, $Q_{p}(s)$ has the same number of zeros and poles, then $\left.M_{Q_{p}}(\omega)\right|_{\omega \rightarrow \infty}=k_{p}$. For the first case, the condition of Lemma 5 is fulfilled. For the second case, it is necessary that $k_{p}<1$. Therefore it can be concluded that if the conditions obtained in Theorem 1 are fulfilled, then there is exactly one anticlockwise encirclement to the point $(-1, \mathrm{j} 0)$. Thus the Nyquist criteria is satisfied ensuring the stability of the closed-loop system as long as the gain $k_{p}$ is well selected.

Remark 1. Following Lemma 6, P-stabilizabilty condition automatically becomes sufficient for the PI-stabilizabilty condition. In this way, similar stability analysis can be performed for the auxiliary system $Q_{P I}(s)$ obtaining the same result.

Remark 2. The magnitude of the unstable delayed system without zeros, decreases monotonically in all the cases, reducing the drawback of stability analysis to the phase condition. The stabilizability analysis becomes more difficult in the present work, due to the presence of additional zero dynamics in the transfer function. As a consequence, condition (11) is derived from the analysis.

Remark 3. In order to design the P controller $C_{p}(s)$, the proportional gain $k_{p}$, can be obtained from the following facts; it is required that $M_{Q_{p}}(\omega)>1$ for some positive frequency, in order to encircle the point $(-1, \mathrm{j} 0)$, that is: $k_{p} \sqrt{\prod_{l=1}^{m}\left(\omega_{c_{1}}^{2}+b_{l}^{2}\right) / \prod_{i=1}^{n+1}\left(\omega_{c_{1}}^{2}+a_{i}^{2}\right)}>1$

Taking into account that $\omega_{c_{1}}=0$, the lower bound of the gain $k_{p}$ can be obtained.

$k_{p}>\prod_{i=1}^{n+1} a_{i} / \prod_{l=1}^{m} b_{l}$

From $M_{Q_{P}}\left(\omega_{c_{2}}\right)<1$, the upper bound for $k_{p}$ can be stated as follows:

$$
k_{p}<\sqrt{\prod_{i=1}^{n+1}\left(\omega_{c_{2}}^{2}+a_{i}^{2}\right) / \prod_{l=1}^{m}\left(\omega_{c_{2}}^{2}+b_{l}^{2}\right)}
$$

The stabilizing proportional gain $k_{p}$ should satisfy:

$$
\frac{\prod_{i=1}^{n+1} a_{i}}{\prod_{l=1}^{m} b_{l}}<k_{p}<\sqrt{\frac{\prod_{i=1}^{n+1}\left(\omega_{c_{2}}^{2}+a_{i}^{2}\right)}{\prod_{l=1}^{m}\left(\omega_{c_{2}}^{2}+b_{l}^{2}\right)}}
$$

Remark 4. The sufficient stability conditions for PD/PID controllers can be obtained with a similar analysis, in the same way that the proof from Theorem 1 has been shown. Consider the system (1) with $H(s)$ in the form shown in (4), $m<n+1$ and the PD/PID controller given by (7)/ (8). Therefore, the associated closed-loop is stable if:

$$
\frac{k_{D} \prod_{l=1}^{m} b_{l}}{\prod_{i=1}^{n+1} a_{i}}>\sqrt{\frac{\left(k_{D}{ }^{2}+\omega^{2}\right) \prod_{l=1}^{m}\left(\omega^{2}+b_{l}{ }^{2}\right)}{\prod_{i=1}^{n+1}\left(\omega^{2}+a_{i}{ }^{2}\right)}}, \forall \omega>0
$$

and

$\tau<\frac{1}{a_{1}}+\sum_{l=1}^{m} \frac{1}{b_{l}}-\sum_{i=2}^{n+1} \frac{1}{a_{i}}+\frac{1}{k_{D}}$

There are two approaches to apply the sufficient conditions (22)-(23). The first proposal consists in choosing, $k_{D}=\min \left(a_{2}, a_{3}, \ldots, a_{n}\right)$. The main idea is to perform a zero-pole cancelation to obtain a better time-delay condition than the $\mathrm{P} / \mathrm{PI}$ controller condition. On the other hand, the second proposal consists in improving 
the time-delay condition following the next methodology: a $k_{D}$ can always be selected such that $\max \left(\phi_{Q_{P D}}(\omega)\right)>-\pi$ if:

$\frac{1}{k_{D}}>\tau-\frac{1}{a_{1}}-\sum_{l=1}^{m} \frac{1}{b_{l}}+\sum_{i=2}^{n+1} \frac{1}{a_{i}}$

From (23) $k_{D}$ can be selected such that the condition (22) is satisfied, that is:

$\overline{k_{D}}=\frac{1}{\tau-\frac{1}{a_{1}}-\sum_{l=1}^{m} \frac{1}{b_{l}}+\sum_{i=2}^{n+1} \frac{1}{a_{i}}}+\varepsilon$

with $\varepsilon$ being a positive real constant.

It can be easily noticed that for a sufficient small $\varepsilon$, one gain $k_{D}$ can be found such that the condition (23) is satisfied. Taking into account (25), the magnitude expression can be written as follows:

$\overline{M_{Q_{P D}}}(\omega)=\sqrt{\frac{\left({\overline{k_{D}}}^{2}+\omega^{2}\right) \prod_{l=1}^{m}\left(b_{l}^{2}+\omega^{2}\right)}{\prod_{i=1}^{n+1}\left(a_{i}^{2}+\omega^{2}\right)}}$

It is necessary to select $k_{D}$ such that $\overline{M_{Q_{P D}}}(0)>\overline{M_{Q_{P D}}}(\omega), \forall \omega$ (equivalent condition to that shown in (22)). The proportional gain $k_{p}$ can be picked into the following interval:

$$
\frac{\prod_{i=1}^{n+1} a_{i}}{k_{D} \prod_{l=1}^{m} b_{l}}<k_{p}<\sqrt{\frac{\prod_{i=1}^{n+1}\left(\omega_{c_{2}}^{2}+a_{i}^{2}\right)}{\left(\omega_{c_{2}}^{2}+k_{D}{ }^{2}\right) \prod_{l=1}^{m}\left(\omega_{c_{2}}^{2}+b_{l}^{2}\right)}}
$$

Section 5 will illustrate that a better time-delay condition with respect to other similar solution taken from the recent related literature can be achieved by employing these last methodologies.

Remark 5. Following the Lemma 6, PDstabilizabilty condition automatically becomes sufficient PID-stabilizabilty condition. In this way, similar stability analysis can be performed for the auxiliary system $Q_{P I D}(\omega)$ obtaining the same result.

\section{2. $H_{\infty}$ Control Design Procedure}

The authors propose the use of the optimum $H_{\infty}$ theory in order to provide optimal gains for PI/
PID controllers with respect to some design specifications. A non-smooth $H_{\infty}$ optimization method is applied to tune the controller parameters.

Figure 3 shows the generalized plant for $H_{\infty}$ mixed sensitivity problem that involves two or more sensitivity functions. Each element is assumed to be LTI, $P(s)$ is a polynomial openloop plant. $W_{e}, W_{u}, W_{d}$ are weights for specifying the system performance, $e_{1}$ and $e_{2}$ are regulated outputs, $y$ is the measured output, $r$ is the reference input, $u$ is the control input, $d$ is the disturbance input and $C_{*}$ is the controller that combines all tunable control parameters. The transfer matrix from $r$ and $d$ can be defined as follows:

$\left[\begin{array}{l}e_{1} \\ e_{2}\end{array}\right]=\left[\begin{array}{cc}W_{e} S & W_{e} S P W_{d} \\ W_{u} C_{*} S & W_{u} T W_{d}\end{array}\right]\left[\begin{array}{l}r \\ d\end{array}\right]$

where $S=\left(1+P C_{*}\right)^{-1}$ is the sensitivity function and $T=C_{*} P S$ is the complementary sensitivity function. The principal result of the $H_{\infty}$ standard problem is: for $\gamma$ as small as possible, find a stabilizing controller $C_{*}(s)$ such that:

$\left\|\begin{array}{cc}W_{e} S & W_{e} S P W_{d} \\ W_{u} C_{*} S & W_{u} T W_{d}\end{array}\right\|_{\infty}<\gamma$

The objective of this research work is to use the $H_{\infty}$ theory and the mixed sensitivity design method to pattern a robust $H_{\infty}$ controller, i.e., to find optimal tunable gains for PI/PID controllers.

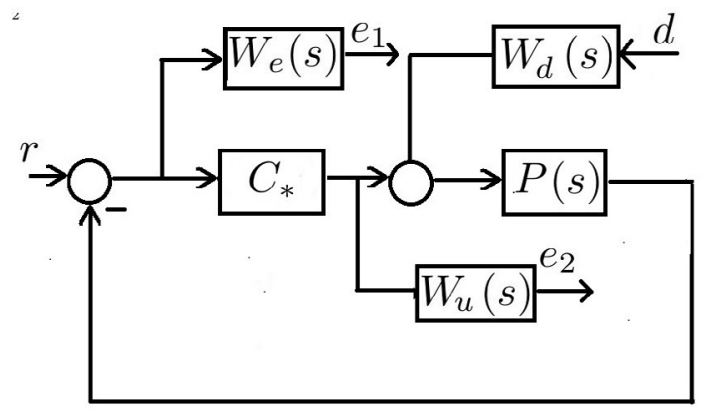

Figure 3. Generalized plant for $H_{\infty}$ mixed sensitivity problem

In other way, the controller $C_{*}(s)$ cannot be designed taking into account $H_{\infty}$ control theory if the delay involved in the plant is treated strictly (Zhang, 1998). When a Padé approximation is introduced, the system (1) becomes:

$H(s) e^{-\tau s} \approx H(s) G_{p}(s)=P(s)$

where $G_{p}(s)$ represents the Padé approximation and $H(s)$ is the delay-free plant. Using (30), 
design techniques developed for systems without delays can be applied and therefore a controller using $H_{\infty}$ theory can be calculated. Therefore, in this work, a Padé approximation is used to perform the $H_{\infty}$ optimization method.

Weighting functions selection. The weighting functions $W_{e}, W_{u}, W_{d}$ are chosen taking into account the basic requirement of mixedsensitivity design (Lundstrom et al., 1991). The main objective of $W_{e}$ is to reduce the error sensitivity in the low-frequency interval for output disturbance rejection. $W_{e}$ is defined as (Skogestad \& Postlethwaite, 2005):

$W_{e}(s)=\frac{1}{M_{s}} \frac{s+\omega_{b} M_{s}}{s+\omega_{b} \varepsilon}$

where $M_{s}$ is the maximum allowable peak for the frequency response of the $S$ and $\omega_{b}$ is the lowest allowable bandwidth of the system.

The principal objective of $W_{u}$ is to ensure the stability of the closed-loop system under diverse operating conditions. $W_{u}$ is defined as follows:

$$
W_{u}(s)=\frac{1}{\varepsilon_{u}} \frac{s+\frac{\omega_{h}}{M_{u}}}{s+\frac{\omega_{h}}{\varepsilon_{u}}}
$$

where $M_{u}$ represents the effort of the controller. $\varepsilon_{u}$ must be chosen as a small constant to ensure a good rejection of the measurement errors. The pulsation $\omega_{h}$ limits the bandwidth. Here, $W_{d}$ is chosen as a constant function in order to ensure the rejection of disturbances.

Augmented plant structure. The augmented plant model can be obtained, as seen in Figure 3. The augmented system contains the nominal system, the weighting functions, the Padé approximation, and the PI/PID controller.

Controller using $H_{\infty}$ structured design. A $H_{\infty}$ structured design method is used to synthesize the controller in order to get a stable solution guaranteeing that the cost function $\gamma$ is minimized. The theory behind is described in (Apkarian \& Noll, 2006). The algorithm iteratively adjusts the parameters of weighting functions until the design objective is satisfied. To apply the $H_{\infty}$ optimization method, it is necessary to provide an initial controller value as a starting point. The initial controller value can be selected taking into account the gains where the system (1) is stable using a P/PD controller. In this way, equations (20), (24) and (26) can be used to select this value.

\section{Examples}

The effectiveness of the proposed methodologies is evaluated by means of two examples.

Example 1: Application of a non-ideal CSTR. This example will show the design method of $\mathrm{P}$ and PI controllers through the following practical example.

Consider the mathematical model for an isothermal CSTR, this example is taken from (Liou et al., 1991). The nonideal mixing is described by the Cholette's model shown in Figure 4.

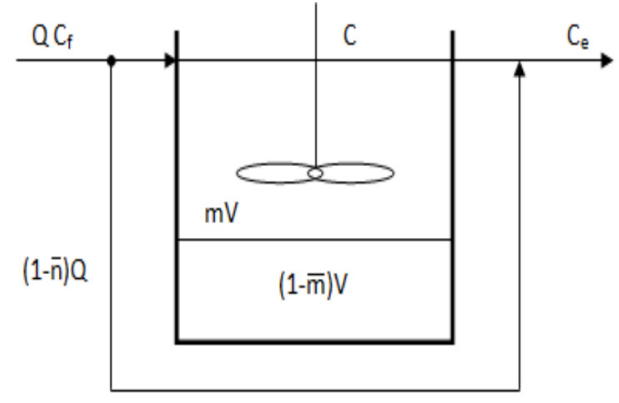

Figure 4. Nonideal CSTR

A simplified model of the dynamics of the process can be described by the following nonlinear differential formulas:

$$
\begin{aligned}
& \frac{d c}{d t}=\left(\frac{\bar{n} Q}{\bar{m} V}\right)\left(C_{f}-C\right)-\frac{k_{1} C}{\left(1+k_{2} C\right)^{2}} \\
& \bar{n} C+(1-\bar{n}) C_{f}=C_{e}
\end{aligned}
$$

The controller variable is the concentration of the reactant in the exit stream $C_{e}$ and the manipulated variable is $C_{f}$ (feed concentration), $C$ is the concentration of the reactant in the wellmixed zone, $\bar{n}$ is the fraction of the reactant feed that enters the zone of perfect mixing and $\bar{m}$ is the fraction of the total volume of the reactor where the reaction occurs. Constants $k_{1}$ and $k_{2}$ describe the reaction rate, and $q$ is the inlet flow rate.

For the present simulation study, $\bar{n}=\bar{m}=0.75$, $k_{1}=10 \mathrm{~s}^{-1}, \quad k_{2}=10(\mathrm{~mol} / \mathrm{l})^{-1}, \quad V=1 \mathrm{l}$. For $C_{f}=3.288 \mathrm{~mol} / \mathrm{l}, \quad C_{e}=1.8 \mathrm{~mol} / \mathrm{l} \quad$ and $C=1.304 \mathrm{~mol} / \mathrm{l}$ are considered. The 
nonlinear model around this nominal operating point taking into account a delay of 20 s gives the next transfer function:

$$
G(s)=\frac{\Delta C_{e}(s)}{\Delta C_{f}(s)}=\frac{2.21(11.13 s+1)}{(98.3 s-1)} e^{-20 s}
$$

The mathematical model equation for a CSTR with nonideal mixing assuming a measurement delay of $20 \mathrm{~s}$ is given by (Liou et al., 1991). From Theorem 1 it follows that the sufficient conditions for $\mathrm{P}$ and PI controllers are satisfied, that is:

$$
\tau=20<\frac{1}{0.0898}+\frac{1}{0.0102}=109.17
$$

For $\mathrm{P}$ controller design, from equation (21) the next range for $k_{p}$ is obtained: $0.1136<k_{p}<0.8035$. A gain $k_{p}=0.3$ is selected. The Nyquist plot of $C_{P}(s) G(s)$ is given in Figure 5 which indicates a stable closed-loop system.

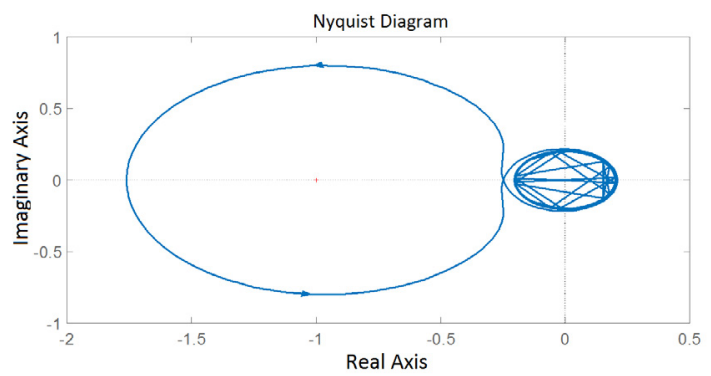

Figure 5. Nyquist diagram of Example 1 using a $P$ controller

The dynamic behaviour of the controlled system is shown in Figure 6.

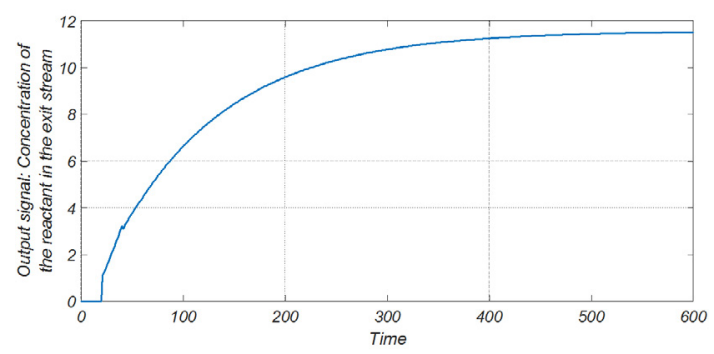

Figure 6. Closed-loop behaviour of Example 1 using a $\mathrm{P}$ controller

To obtain disturbance rejections and reference tracking, the optimal gains for a PI controller are provided. Consider the PI controller $C_{P I}(s)$. Using the methodology presented in Section 5, taking into account a Padé-approximation of third order, $W_{e}(s)$ is considered with the following parameters: $M_{s}=1.2, \omega_{b}=0.1, \varepsilon=0.0103$. The weighting function $W_{u}(s)$ is considered with the parameters: $M_{u}=10, \omega_{h}=10$, $\omega_{h}=0.0001$ and $W_{d}(s)=0.001$, the initial controllers values selected are $k_{i}=0.001$ and $k_{p}=0.1$. The gains obtained by the optimization algorithm are $k_{i}=0.02$ and $k_{p}=0.55$ which are used in simulation experiments. Additionally, a conventional PI controller (without optimization) used to stabilize the plant shown in (35) is presented in order to make a comparison and show the advantages of $H_{\infty}$ structured design method. The gains values for the simulation of conventional PI are $k_{p}=0.205$ and $k_{i}=4.75 e^{-8}$.

The dynamic behavior of the closed-loop system using a PI controller is shown in Figure 7. The blue line indicates the simulation of the conventional PI controller and the red line indicates the simulation of the proposed strategy. The results obtained for the optimized PI controller do not overshoot $20 \%$ this value being lower than the response of the conventional PI controller (overshoot of 120\%). The PI $H_{\infty}$ presents a faster response compared with the conventional PI controller. Both controllers have the same performance tracking of the reference. The analysis results confirm the validity of the proposed control technique.

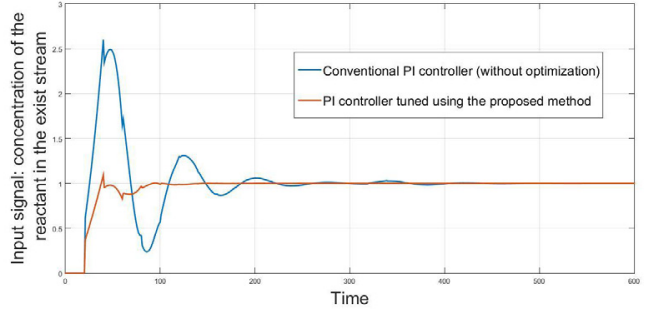

Figure 7. Closed-loop behaviour of Example 1 using a conventional PI controller and the proposed method

Example 2. The results about PD/PID stabilization shown in this paper are compared with alternative strategies taken from the recent related literature.

Take the example proposed in (Lee \& Wang, 2010).

$G(s)=\frac{(s+0.833)}{(s-1)(s+0.909)(s+5)(s+5)} e^{-\tau s}$

The maximum time-delay allowed to stabilize the system given by (37) in (Lee \& Wang, 2010) using a PD/PID controller is $\tau<1.039$, but the authors take into account a delay $\tau<0.1$ to show 
the feasibility of this proposal. Now, consider the system shown in (37) with $\tau=1.04$, the stability condition proposed in (Lee \& Wang, $2010)$ is violated. In this second proposal, the methodology shown in Remark 4 is used in order to solve the problem.

For PD controller design, using the equation (25) a $\varepsilon=2.2212$ and a gain $k_{D}=2.273$ are selected such that the condition (22) is satisfied. From equation (27) the next range for $k_{p}$ is obtained: $12.0255<k_{p}<12.971$. A gain $k_{p}=12.3$ is selected. The Nyquist plot of $C_{P D}(s) G(s)$ is given in Figure 8 which indicates a stable closedloop system.

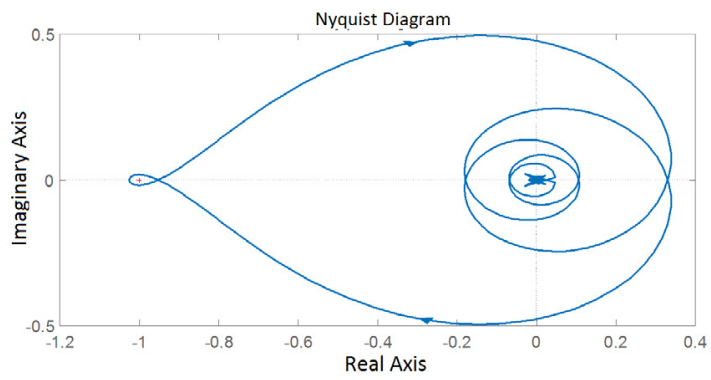

Figure 8. Nyquist diagram of Example 2 using a PD controller

\section{REFERENCES}

1. Apkarian, P. \& Noll, D. (2006). Nonsmooth optimization for multidisk synthesis, European Journal of Control, 12(3), 229-244.

2. De Paor, A. M. \& O'Malley, M. (1989). Controllers of Ziegler-Nichols type for unstable process with time delay, International Journal of Control, 49(4), 1273-1284.

3. Doyle, J. C., Glover, K., Khargonekar, P. P. \& Francis, B. A. (1989). State-space solutions to standard $\mathrm{H}_{2}$ and $\mathrm{H}_{\infty}$ control problems, IEEE Transactions on Automatic Control, 34(8), 831-847.

4. Kharitonov, V. L. (1998). Robust stability analysis of time delay systems: A survey. In 5th IFAC conference on Systems Structure and Control 1998 (SSC'98), Nantes, France (pp. 1-12).

5. Kwak, H. J, Sung S. W. \& Lee, I-B. (2000). Stabilizability conditions and controller design for unstable processes, Chemical Engineering Research \& Design, 78(4), 549-556.

\section{Conclusion}

This paper addresses the stabilization and control problem of a particular kind of linear delayed systems containing zero dynamics in the input-output path. The sufficient conditions needed to ensure the stabilization of this class of systems are provided. The results consider the employment of P, PI, PD, and PID controllers. A frequency domain analysis is used to demonstrate the principal results. Using $H_{\infty}$ theory the controller parameters for PI/ PID controllers are tuned taking into account a non-smooth $H_{\infty}$ optimization method. Finally, examples by numerical simulations are provided in order to confirm the feasibility of the proposed controllers.

6. Lee, S. C., Wang, Q.-G. \& Xiang, C. (2010a). Stabilization of all-pole unstable delay processes by simple controllers, Journal of Process Control, 20(2), 235-239.

7. Lee, S. C.\& Wang, Q.-G. (2010). Stabilization conditions for a class of unstable delay processes of higher order, Journal of the Taiwan Institute of Chemical Engineers, 41(4), 440-445.

8. Lee, S. C., Wang, Q.-G. \& Le, B. N. (2010b). Stabilizing control for a class of delay unstable processes, ISA Transactions, 49(3), 318-325.

9. Liou, C.-T. \& Chien, Y.-S. (1991). The effect of nonideal mixing on input multiplicity in a CSTR, Chemical Engineering Science, 46(8), 2113-2116.

10. Liu, T., Zhang, W.\& Gu, D. (2005). Analytical design of two-degree-of-freedom control scheme for open-loop unstable processes, Journal of Process Control, 15(5), 559-572. 
11. Lundstrom, P., Skogestad, S. \& Wang, Z. Q. (1991). Performance weight selection for H-infinity and $\mu$ control methods, Transactions of the Institute of Measurement and Control, 13(5), 241-252.

12. Marquez-Rubio, J. F., del-Muro-Cuéllar, B., Velasco-Villa, M. \& Álvarez-Ramírez, J. (2010). Control based in an observer scheme for first-order systems with delay, Revista Mexicana de Ingeniería Química, 9(1), 43-52.

13. Nyquist, H. (1932), Regeneration theory, The Bell System Technical Journal, 11(1), 126-147.

14. Qing-Chang, Z. (2006). Robust Control of Time-Delay Systems. London: Springer-Verlag.

15. Seshagiri, A. R., Rao, V. S. R. \& Chidambaram, M. (2007). Simple analytical design of modified smith predictor with improved performance for unstable firstorder plus time delay processes, Industrial \& Engineering Chemistry Research, 46(13), 4561-4571.

16. Silva, G. J., Datta, A. \& Bhattacharyya, S. (2004). PID Controllers for Time-Delay Systems. Control Engineering Series, Birkhäuser.

17. Skogestad, S. \& Postlethwaite, I. (2005). Multivariable feedback control: Analysis and Design, $2^{\text {nd }}$ edition. Wiley-Interscience.
18. Smith, J. M. (1957). Closer Control of Loops with Dead Time, Chemical Engineering Progress, 53(5), 217-219.

19. Vázquez, C. D. Marquez-Rubio, J. F. \& del Muro, B. (2017) Stability conditions for unstable high order systems with minimumphase zeros plus time delay. In Congreso Nacional de Control Automático (AMCA), Nuevo León, México (pp. 1-6).

20. Wu, L, Lam, H.-K., Zhao, Y. \& Shu, Z. (2015). Time-Delay Systems and Their Applications in Engineering 2014, Mathematical Problems in Engineering, 2015, Article ID 246351, 3 pages.

21. Xiang, C., Wang, Q.-G., Lu, X., Nguyen, L. \& Lee, T. H. (2007). Stabilization of second order unstable delay processes by simple controllers, Journal of Process Control, 17(8), 675-682.

22. Xiang, L. (2006). Advances in PID, Smith and Deadbeat Control. PHD Thesis. Singapore: Department of ECE, National University of Singapore.

23. Zhang, W.D.(1998). Analytical design methods for process control. Postdoctoral Research Report. Shanghai Jiaotong University. 